\title{
The world's last best hope: el excepcionalismo americano y la política exterior de Estados Unidos en la era Obama
}

\section{The World's Last Best Hope: American Exceptionalism and US Foreign Policy in Obama’s Era}

\author{
Manuel Iglesias Cavicchioli ${ }^{1}$ \\ Universidad Loyola Andalucía (España)
}

Recibido: 29-11-17

Aprobado: 28-02-18

\section{Resumen}

Desde la llegada al poder de Barack Obama, el excepcionalismo americano se ha situado en el centro del debate político en estrecha conexión con el debate en torno al declive internacional de EE.UU. El presente artículo pretende ofrecer un estudio del excepcionalismo tanto desde el punto de vista teórico como desde su impacto más reciente en la política exterior de EE.UU. A tal efecto, procederemos a desentrañar sus principales aspectos conceptuales, analizando las distintas interpretaciones del mismo en el marco de las principales doctrinas de política exterior estadounidense. Sobre esta base teórica, nos detendremos en la influencia de la idea excepcionalista en la práctica de la política exterior de la Administración Obama, denunciada por sus más acerbos críticos como antiexcepcionalista y, por inmediata extensión, como antiamericana.

Palabras-clave: excepcionalismo americano, política exterior estadounidense, Administración Obama.

\footnotetext{
${ }^{1}$ (miglesias@uloyola.es) Profesor de Relaciones Internacionales de la Universidad Loyola Andalucía. Doctor en Relaciones Internacionales por la Universidad Complutense de Madrid (UCM) y Máster en Estudios Internacionales por la Escuela Diplomática de España. Ha sido becarioinvestigador FPU y Profesor Ayudante en la Facultad de Ciencias Políticas y Sociología de la UCM. Entre sus publicaciones recientes destacan las monografías Aznar y los "neocons": el impacto del neoconservadurismo en la política exterior de España, prólogo de Celestino del Arenal, Barcelona, Huygens, 2017 y La visión neoconservadora de las relaciones internacionales y la política exterior de Estados Unidos, prólogo de Antonio Remiro Brotons, Barcelona, Huygens, 2016.
} 


\begin{abstract}
Since Barack Obama came to power, American exceptionalism has been in the center of the political debate in close connection with the issue of US decline in the world stage. This article will deal with American exceptionalism from both a theoretical perspective and from its impact in nowadays US foreign policy. To this end, we will proceed to elucidate the main conceptual aspects of American exceptionalism, analyzing its different interpretations within the framework of the main US foreign policy doctrines. On this theoretical basis, we will analyze the practical influence of the excepcionalist idea in Obama administration's foreign policy, which has been denounced by its harshest critics as anti-exceptionalist and, by extension, as un-American.
\end{abstract}

Key-words: American exceptionalism, US foreign policy, Obama administration.

\title{
1. Introducción: las crisis del excepcionalismo y su persistente relevancia en la política americana
}

Cuando se habla del excepcionalismo americano, se está haciendo referencia a un tema de gran relevancia en la política, en la sociedad y en la cultura de EE.UU. a lo largo de toda su historia como nación ${ }^{2}$. El excepcionalismo ha experimentado dos grandes crisis. La primera de ellas, y seguramente la más grave, fue la vivida en los años 70 del pasado siglo con la larga implicación y final derrota estadounidense en la guerra de Vietnam, la política de distensión de Nixon-Kissinger y la presidencia de Carter. Aunque entonces el sociólogo Daniel Bell llegó a proclamar "el fin del excepcionalismo americano" (citado en: McCrisken, 2003: 2 y 3), la llegada a la Casa Blanca de Ronald Reagan, con un discurso de grandeza nacional encaminado a recuperar el orgullo americano, supuso un reimpulso sustancial para el excepcionalismo americano (Barone, 2013. Hayward, 2013). El fin de la Guerra Fría y la unipolaridad resultante, con las presidencias de Bush y Clinton, supusieron la continuidad del mensaje excepcionalista en la política exterior estadounidense, si bien con importantes matices diferenciadores en cada caso (McCrisken, 2003: 154-158 y 180-182).

El 11 de septiembre (11-S) causó un enorme impacto en la opinión pública y la política estadounidense, poniendo fin a la sensación de invulnerabilidad territorial que había acompañado a EE.UU. a lo largo de su historia. Se puede afirmar que el auge excepcionalista acaecido por la reacción de la Administración Bush ante el 11-S, con la vuelta del espíritu de cruzada a tiempos álgidos de la

\footnotetext{
${ }^{2}$ Para el impacto del excepcionalismo en la cultura americana, véase: Söderlind, Carson (eds.), 2011.
} 
Guerra Fría, la implicación estadounidense en Afganistán e Irak, y la posterior elección de Barack Obama constituyen un nuevo periodo de crisis — la segunda crisis- del excepcionalismo americano.

La enorme polarización política existente en EE.UU. durante el mandato de Obama, en el periodo de enfrentamiento partidista más agudo desde el fin de la Guerra Fría, ha llevado a que el excepcionalismo se convierta en uno de los elementos cruciales del debate político estadounidense. La llegada de Obama al poder, de hecho, ha supuesto que el debate en torno al excepcionalismo americano haya desbordado los límites del mundo académico, que es donde habitualmente se ha desarrollado el mismo, y se haya convertido en un asunto destacado de la batalla política y electoral entre los partidos Republicano y Demócrata (Dunn, 2013: 12). Ello ha motivado el empleo del excepcionalismo como arma arrojadiza ideológica, provocando ciertas distorsiones y tergiversaciones conceptuales que es necesario aclarar. Así, desde sectores liberales se han lanzado críticas contra el excepcionalismo identificándolo con la política exterior de George W. Bush, como si fuera una idea inventada por éste o por los neoconservadores; mientras que desde sectores conservadores las críticas han ido dirigidas a tachar la política exterior (e interior) de Obama como antiexcepcionalista y antiamericana.

Conviene señalar en este punto que el excepcionalismo americano tiene una doble vertiente: la interior, 'doméstica' o nacional; y la exterior o internacional. Se trata de planos indudablemente interconectados pero entre los que existe una diferencia material clara que debe ser mantenida a efectos analíticos y explicativos. En el ámbito interno, el excepcionalismo alude, fundamentalmente, a tres temas: el papel de la religión en la sociedad y en la política estadounidenses; el papel del Estado en la economía y en la sociedad; $\mathrm{y}$, finalmente, la cuestión inmigratoria (Brooks, 2013). En cuanto al ámbito exterior, la cuestión central del excepcionalismo alude al papel de EE.UU. en las relaciones internacionales, a su misión en el mundo.

Este trabajo, como su propio título indica, se centrará únicamente en este último aspecto: la incidencia del excepcionalismo en la política exterior americana. Para ello el siguiente apartado se dedicará a realizar las precisiones conceptuales necesarias sobre el excepcionalismo, desgranando sus aspectos materiales e inmateriales y analizando sus principales aspectos teóricos. Una vez realizada esta necesaria tarea inicial, se procederá a analizar las distintas visiones del excepcionalismo desde las principales doctrinas de política exterior americanas. Ello permitirá distinguir el excepcionalismo de conceptos políticos concomitantes como el aislacionismo, que se refiere a una tradición o doctrina de política exterior estadounidense que posee su propia interpretación del excepcionalismo ${ }^{3}$. Se prestará atención, finalmente, a la praxis de la política

\footnotetext{
${ }^{3}$ Aunque tanto el excepcionalismo como el aislacionismo son ideas sociopolíticas típicamente
} Primer semestre de 2019. Pp. 161-185. ISSN 1575-6823 e-ISSN 2340-2199 doi: 10.12795/araucaria.2019.i41.08 
exterior americana del siglo XXI y, en concreto, en el papel del excepcionalismo en la política exterior de Barack Obama, con una necesaria referencia a su predecesor, George W. Bush.

\section{Aspectos conceptuales: poder e ideas en el excepcionalismo americano}

El excepcionalismo americano surge con mucha anterioridad al nacimiento de EE.UU. como Estado independiente. Los elementos seminales del mismo se pueden hallar en el célebre sermón de John Winthorp, frente a la bahía de Massachusetts, a bordo del barco Arbella, donde aparece la no menos célebre imagen bíblica de "la ciudad en la cima"(Hodgson, 2009: 1) ${ }^{4}$, verdadero lugar común del excepcionalismo. No obstante, la noción aparece como tal, en el sentido extendido actualmente, gracias a Alexis de Tocqueville, quien en su obra clásica La democracia en América emplea por primera vez el término 'excepcional' para caracterizar al pueblo estadounidense (Tocqueville, 2002: 51. Lipset, 2000).

Para avanzar adecuadamente en su estudio, es necesario discernir dos dimensiones fundamentales en el excepcionalismo americano: su dimensión material u objetiva, por un lado; y su dimensión espiritual, subjetiva o ideacional, por otro. Se trata de dimensiones que, en todo caso, guardan, como enseguida se verá, una interrelación clara y estrecha.

La primera de ellas se refiere al poder duro, principalmente militar, aunque también económico, de EE.UU., así como a su situación geográfica. En cuanto al poder estadounidense, es sabido que el mismo ha experimentado enormes cambios desde que EE.UU. alcanzara su independencia en 1776. El excepcionalismo, como se ha apuntado, es una noción anterior a esta fecha y, por supuesto, muy anterior a la llegada de EE.UU. al estatus de superpotencia mundial tras la Segunda Guerra Mundial. Por lo tanto, el poder estadounidense no puede ser considerado ab initio la causa del excepcionalismo americano, aunque indudablemente, en perspectiva histórica, ha influido en la orientación y aplicación del mismo. En este sentido, es indudable que tras la Segunda Guerra Mundial el excepcionalismo se ha convertido en un instrumento ideológico-discursivo preferente para justificar, legitimar y tratar de perpetuar la acción global y la hegemonía estadounidenses, encarnada en la idea del 'siglo americano' (Bacevich, 2012).

estadounidenses que están estrechamente relacionadas, la primera posee una mayor amplitud y transversalidad que la segunda. El excepcionalismo nunca ha sido una doctrina de política exterior, pero sí una idea influyente en las distintas corrientes de política exterior de EE.UU. y susceptible de diversas interpretaciones.

${ }^{4}$ City upon a hill, en la expresión original en inglés 
Por otro lado, está fuera de discusión que la situación geográfica ha sido determinante en el papel de EE.UU. en el mundo. La lejanía física de Europa no sólo facilitó la propia independencia estadounidense, sino que propició el desarrollo del proyecto político americano al margen de la injerencia de las potencias europeas (Heclo, 2013: 30). Es incuestionable, pues, que, a partir de la realidad geográfica estadounidense, se construye el excepcionalismo como idea, aunque, como veremos, esta realidad ha perdido importancia conforme el papel de EE.UU. en el mundo se ha expandido y ha llegado a tener alcance global.

La segunda dimensión mencionada alude a aspectos inmateriales del excepcionalismo como las ideas y las creencias que conforman la cultura política y la identidad nacional del país. En efecto, el excepcionalismo no se limita a los aspectos materiales citados ni se puede explicar únicamente a través de los mismos, sino que es fundamentalmente una idea, una creencia (McCrisken, 2003: 4-6). Como ha señalado McCrisken (2003: 5) ${ }^{5}$, en EE.UU. existe una narrativa de ser una nación excepcional, diferente al resto del mundo, que se ha desarrollado a lo largo de la historia estadounidense. Se podría hablar, por ende, de la dimensión mesiánica del excepcionalismo americano, sin la cual es imposible explicar y entender la importancia de este fenómeno en la política exterior estadounidense.

El excepcionalismo es, pues, una creencia que da lugar a una auto-imagen de los propios estadounidenses, arraigada con fuerza tanto en sus élites políticas como en su opinión pública, según la cual EE.UU. sería una nación cualitativamente diferente al resto del mundo, una nación única. De este modo, diferencia y unicidad son elementos definitorios del excepcionalismo (Ceaser, 2013: 16). El excepcionalismo implica también superioridad, no sólo material, sino fundamentalmente (y desde luego, inicialmente) moral de los valores americanos (McCrisken, 2003: 1). Esta superioridad, aunque subyace en todas las visiones excepcionalistas actuales, se hace explícita particularmente en alguna de ellas, como se verá en el caso neoconservador, donde se ha formulado de manera abierta y sin ambages. La auto-imagen excepcionalista opera una división radical entre el Nuevo Mundo, que encarna EE.UU., y el Viejo Mundo, encarnado en un primer momento por Europa, de modo que el excepcionalismo podría ser definido como "la autoimagen separatista de América" (Nau, 2002: 1).

En materia de política exterior, que es la que concierne a este artículo, la dimensión espiritual del excepcionalismo se refiere, esencialmente, a la misión de EE.UU. en el mundo, esto es, a su papel como agente defensor y difusor de

\footnotetext{
${ }^{5}$ La idea de excepcionalismo no nace en el vacío, sino que toma los elementos materiales señalados para conformarse, de ahí la interrelación señalada entre ambas dimensiones, pero el excepcionalismo, como concepto ideacional trasciende ampliamente esa base material u objetiva.
}

Araucaria. Revista Iberoamericana de Filosofia, Politica, Humanidades y Relaciones Internacionales, año $21, \mathrm{n}^{\circ} 41$. Primer semestre de 2019. Pp. 161-185. ISSN 1575-6823 e-ISSN 2340-2199 doi: 10.12795/araucaria.2019.i41.08 
los valores de la democracia liberal (Ceaser, 2013: 17 y 18. Heclo, 2013: 33). Se trata de la creencia de que EE.UU. es una nación especial con un papel especial que jugar en la historia de la humanidad (McCrisken, 2003: 8). La idea de misión, de destino especial, en efecto, es central en el mito fundacional de EE.UU., y está presente ya en el aludido discurso de Winthorp. Es indudable que en la política exterior estadounidense ha prevalecido la idea de que EE.UU. tiene una misión que cumplir en el mundo y que ésta es la de defender y difundir sus valores, sus principios de gobierno, liberando, así, a los pueblos y naciones oprimidas.

Aparejada a la unicidad estadounidense y a su sentido de misión, se halla la creencia excepcionalista de que EE.UU., a diferencia de otras grandes naciones, no está destinado a "subir y caer", escapando, así, a las leyes de la Historia (McCrisken, 2003: 10). En esta idea subyace, sin duda, el típico optimismo excepcionalista, con su característica fe en el progreso de la Humanidad de mano de EE.UU. (Heclo, 2013: 35).

Ahora bien, surge aquí una bifurcación clara en la creencia excepcionalista que permite discernir dos líneas dentro del excepcionalismo en cuanto a cómo debe cumplir su misión EE.UU.: en primer lugar, una línea 'redencionista' (Heclo, 2013: 34) — también denominada 'vindicacionista' (Monten, 2005: 123) o 'misionera' (McCrisken, 2003: 2) — que ve a EE.UU. como "nación redentora" que, por lo tanto, ha de llevar a cabo una política intervencionista dirigida a difundir activamente sus valores para transformar, así, el Viejo Mundo; y, en segundo término, otra línea 'ejemplarista' (McCrisken, 2003: 11. Monten, 2005: 123. Heclo, 2013: 33), también llamada 'aislacionista' (Kissinger, 2010: 10), que preconiza que EE.UU. debe ser un faro, un ejemplo de libertad para el resto del mundo, permaneciendo apartado de sus problemas.

La línea redencionista del excepcionalismo americano tiene en la llamada doctrina del Destino Manifiesto uno de sus principales hitos teóricos. Surgida a mediados del siglo XIX, esta doctrina formula la idea preexistente de que EE.UU. es un pueblo elegido por la Providencia y que tienen que cumplir una misión histórica, un destino manifiesto, en el mundo (Weinberg, 1968. Stephanson, 1996). Esta doctrina recoge, en consecuencia, la narrativa de destino especial que se había ido desarrollando desde el siglo XVII (Brooks, 2013: 63 y 64). De este modo, el Destino Manifiesto no puede ser entendido sino como una doctrina subsidiaria del excepcionalismo americano, una articulación de éste en su dimensión exterior: la singularidad estadounidense que proclama el excepcionalismo requiere una misión a la altura de sus cualidades morales como la que proporciona el Manifest Destiny.

En el marco de esta línea o rama redencionista, cabe, a su vez, realizar una distinción entre una línea más dura, seguida por los neoconservadores, y otra más blanda, seguida por los internacionalistas liberales. Sobre ella se volverá en el siguiente apartado. 
Por otro lado, hay que referirse a la ya mencionada línea ejemplarista del excepcionalismo americano, según la cual EE.UU. debe ser un ejemplo para el resto del mundo. Como ha señalado Spanier: "Desde el comienzo mismo de su vida nacional, los norteamericanos profesaron una fuerte creencia en lo que consideraban su destino: extender, por el ejemplo, la libertad y la justicia social para todos y apartar a la humanidad del mal camino, conduciéndola hacia la Nueva Jerusalén terrestre" (Spanier, 1991: 18). Según el excepcionalismo ejemplarista, EE.UU. debe luchar por perfeccionar su propio modelo sociopolítico tanto como sea posible sin interferir en los problemas de otros países, ya que la intervención en el extranjero no sólo puede ser negativa para quien la reciba sino que puede ser contraproducente para el propio EE.UU. (McCrisken, 2003: 11).

En cuanto a la influencia de ambas líneas, se puede afirmar que, en términos generales, el ejemplarismo ha dominado el excepcionalismo y la política exterior de EE.UU. hasta la Segunda Guerra Mundial, mientras que el redencionismo lo ha hecho a partir de entonces (McCrisken, 2003: 13).

La existencia de estas dos ramas dentro del excepcionalismo ha de ser analizada con mayor profundidad para conocer con la máxima precisión posible su papel e influencia en la política exterior americana. A tal efecto, se estudiarán las principales doctrinas de política exterior estadounidense y del lugar que en ellas ocupa el excepcionalismo. Ello permitirá matizar y precisar las distintas interpretaciones de las dos líneas excepcionalistas señaladas e introducir la crítica de una escuela fundamental en la política exterior estadounidense como es la escuela realista.

\section{Visiones excepcionalistas: el excepcionalismo en las doctrinas de política exterior de EE.UU.}

Como se acaba de señalar, no existe una única concepción del excepcionalismo americano, es más, como se verá, no todas las doctrinas de política exterior estadounidenses son excepcionalistas. Para este cometido se tomarán en cuenta las cuatro doctrinas de política exterior americana que consideramos más representativas: el internacionalismo liberal, el realismo, el neoconservadurismo y el aislacionismo.

\subsection{Internacionalismo liberal}

El internacionalismo liberal, o liberalismo internacionalista, nace en EE.UU. como doctrina de política exterior de la mano del presidente Woodrow Wilson, teórico y practitioner del mismo. El wilsonianismo viene a ser una 
adaptación americana del llamado internacionalismo grociano, debidamente pasado por el tamiz del excepcionalismo estadounidense. No se puede entender el excepcionalismo americano en el siglo XX y en el XXI sin la contribución teórica y práctica del presidente Wilson y de la doctrina internacionalista liberal. Wilson es el presidente excepcionalista por excelencia, "la encarnación del excepcionalismo americano" (Kissinger, 2010: 39).

Wilson pretendía superar la política de poder europea a través de un nuevo orden internacional basado en una comunidad de poder (o principio de seguridad colectiva) en lugar de en el tradicional equilibrio de poder que había imperado hasta entonces en Europa (Kissinger, 2010: 43-48). En este sentido, su proyecto de crear una League of Nations como eje del sistema internacional perseguía una transformación sustancial de los fundamentos que habían regido la política internacional hasta ese momento, con la finalidad idealista de erradicar la guerra de las relaciones internacionales (Knock, 1995). De este modo, el internacionalismo liberal pretende transformar el Viejo Mundo a partir de la acción americana, o dicho de otro modo, pretende poner el poder estadounidense al servicio de la Humanidad, afirmando la universalidad de los valores americanos.

Es indudable que el sentido de misión del Destino Manifiesto está presente como un distintivo constante en el pensamiento wilsoniano. Ahora bien, es preciso subrayar que, a nuestro entender, Wilson imprime a la doctrina del Destino Manifiesto un alcance verdaderamente mundial del que carecía hasta entonces: EE.UU. rompe la separación hemisférica que definía al aislacionismo, da un salto cualitativo a la escena mundial y predica un intervencionismo de alcance global. Para Wilson, EE.UU. tenía el deber de hacer del mundo "un lugar seguro para la democracia", de modo que su misión tiene como objetivo no ya limitarse a ser el faro, el ejemplo para el resto del mundo, sino transformar el funcionamiento del sistema internacional, adecuándolo a los valores estadounidenses de libertad y democracia.

Aunque el proyecto wilsoniano fracasó en el periodo de entreguerras y ni siquiera logró convencer entonces al Senado americano, la implicación de EE.UU. como principal impulsor y arquitecto de las instituciones internacionales surgidas tras la Segunda Guerra Mundial revelan la influencia clara del internacionalismo liberal en la práctica de la política exterior americana. Para los realistas, las ideas wilsonianas han calado tan profundamente en la política exterior de EE.UU. que se han convertido en su principal fuente teórica (Kissinger, 2010: 24).

En la actualidad, cabría distinguir la existencia de dos sectores dentro del internacionalismo liberal de tradición wilsoniana: los llamados 'halcones liberales ${ }^{6}$ o internacionalistas intervencionistas, por un lado, y los llamados

\footnotetext{
${ }^{6}$ Liberal hawks, en la expresión original en inglés.
} 
globalistas o transnacionalistas, por otro. Los primeros defienden la necesidad de emplear el poder militar americano para defender los derechos humanos, impulsando así la injerencia humanitaria. Han tenido una notable influencia durante la presidencia de Clinton, impulsando la guerra de Kosovo bajo la consigna de impedir la limpieza étnica del gobierno serbio en aquella parte de su territorio. Asimismo, este sector del liberalismo internacionalista defendió la guerra de Irak de 2003 y, pese a la constatación de que Irak no poseía Armas de Destrucción Masiva, avaló su carácter benéfico al considerar que logró acabar con un dictador que violaba sistemáticamente los derechos humanos de su población. En este sentido, este sector liberal de línea dura converge con la doctrina neoconservadora a la que luego se hará referencia (Tovar Ruiz, 2014: 149).

Los globalistas americanos, por su parte, se centran más en la dimensión institucionalista y legalista del wilsonianismo, pero adaptándola a la era de la globalización, y de este modo, defienden conceptos liberales herederos de la idea de gobierno mundial como la gobernanza global. Los globalistas ponen en cuestión aspectos determinantes del excepcionalismo como el excepcionalismo constitucional y, sin duda, desacralizan la soberanía estadounidense al defender concepciones poswestfalianas de la soberanía como la soberanía responsable (Fonte, 2012). De ahí que desde el sector excepcionalista neoconservador se considere al globalismo o al progresismo transnacionalista como una doctrina debilitadora de la idea excepcionalista o incluso una amenaza a la soberanía democrática americana (Fonte, 2012). En todo caso, los globalistas americanos buscan que el liderazgo estadounidense se ejerza a través de las instituciones globales, convirtiendo de este modo la gobernanza global en un mecanismo de defensa y difusión de los intereses y valores estadounidenses. De este modo, el globalismo aboga por un excepcionalismo blando, por una visión suave del redencionismo, en el sentido de que no hace del poder militar americano su eje principal, sino que incide en la capacidad transformadora del mundo de EE.UU. a través de las instituciones y normas internacionales.

\subsection{Realismo}

Los realistas estadounidenses afirman que el carácter excepcional de EE.UU. radica en el poder militar que posee desde el fin de la Segunda Guerra Mundial (Ignatieff, 2005: 11) ; $^{7}$ es decir, analizan el excepcionalismo únicamente

\footnotetext{
7 Cuando aquí se emplean los términos 'realismo' y 'realistas' tout court se hace en un sentido amplio como sinónimo de la llamada 'escuela realista', que engloba a distintas teorías que comparten una serie de principios básicos en torno a la política internacional. En este apartado, se verá la manera en que destacados autores pertenecientes al realismo político y al neorrealismo se han posicionado sobre el excepcionalismo. En todo caso, no es el objetivo de este artículo detenerse en las diferencias que existen entre estas teorías realistas, sino sólo constatar que las mismas sostienen ideas muy similares acerca del excepcionalismo.
}

Araucaria. Revista Iberoamericana de Filosofia, Politica, Humanidades y Relaciones Internacionales, año $21, \mathrm{n}^{\circ} 41$. Primer semestre de 2019. Pp. 161-185. ISSN 1575-6823 e-ISSN 2340-2199 doi: 10.12795/araucaria.2019.i41.08 
en su dimensión material. Por consiguiente, rechazan por completo la aludida dimensión mesiánica del excepcionalismo, toda vez que niegan que EE.UU. sea una nación cualitativamente distinta al resto de naciones, y consideran, en cambio, que el excepcionalismo es un "mito" (Hodgson, 2009. Walt, 2011). De este modo, los realistas niegan que EE.UU. deba tener una misión histórica consistente en luchar contra la tiranía y difundir su modelo sociopolítico por el mundo, sea por la vía ejemplarista, sea, de manera aún más clara, por la vía redencionista. Como ha señalado Ceaser, la alternativa antiexcepcionalista al sentido de misión es el realismo, que preconiza una política exterior sin sentido de misión, de modo que realismo y misión son nociones antitéticas (Ceaser, 2013: 19).

El realismo es, por ende, antiexcepcionalista en un aspecto fundamental de la creencia excepcionalista como es su sentido de misión. No hay que olvidar que el realismo preconiza una escisión clara entre mundo interno y mundo internacional: en virtud del principio realista de doble moralidad, los principios morales que rigen en el ámbito interno no son aplicables en abstracto al ámbito internacional (Morgenthau, 1986: 21). Por ello, los realistas han sido siempre críticos con el mesianismo wilsoniano y han mantenido y mantienen una enconada polémica con los neoconservadores, manifiesta en el debate en torno a la guerra de Irak (Rosen (ed.), 2005. Mearsheimer, 2005), y que continúa hasta el presente.

Los realistas políticos americanos han rechazado el mesianismo propio del excepcionalismo americano de manera palmaria. En su obra clásica Politics among Nations, Morgenthau advierte que "el realismo político se niega a identificar las aspiraciones morales de una nación en particular con los preceptos morales que gobiernan el universo" (Morgenthau, 1986: 22). Henry Kissinger, por su parte, ha sido siempre un gran crítico del wilsonianismo y sus implicaciones misioneras, rechazando, por ende, que la difusión de la democracia deba convertirse en misión de EE.UU. y huyendo "del sentido de cruzada desde un punto de vista ideológico” (Arenal, 1990: 145).

Distinto es el caso de Reinhold Niebuhr y George Kennan, que introducen fuertes componentes morales e ideológicos en sus teorías realistas para justificar la política exterior de EE.UU. en los inicios de la Guerra Fría (Arenal, 1990: 132, 133 y 140-141). En cuanto al primero, considerado el padre del realismo americano, es cierto que Niebuhr, como todos los realistas, rechaza de plano el espíritu de cruzada que impregna la concepción redencionista del excepcionalismo (Thompson, 2007), pero estima en cambio, a diferencia de realistas clásicos como Morgenthau, que la política exterior americana no puede estar privada de elementos morales. Para Niebuhr, EE.UU. no ha de embarcarse en cruzadas democratizadoras por el mundo, pero su política exterior sí que ha de reflejar su carácter democrático. En este sentido, es posible afirmar que 
Niebuhr sí admite que EE.UU. es un país diferente y que esa diferencia ha de estar presente en su política exterior. En definitiva, el realismo niebuhriano rechaza, como todas las teorías realistas, el excepcionalismo redencionista, sea wilsoniano o neoconservador, pero admitiría, de manera implícita, una suerte de ejemplarismo realista, distinto, en todo caso, del aislacionista, toda vez que Niebuhr censura el aislacionismo y defiende la implicación plena de EE.UU. en la política internacional (Arenal, 1990: 133).

Mucho más recientemente, autores neorrealistas como Walt y Bacevich han seguido los pasos de los realistas clásicos y se han significado por atacar con dureza el excepcionalismo americano, refutando su carácter mesiánico. Niegan que EE.UU. sea cualitativamente excepcional y que tenga cualquier tipo de misión especial que cumplir en el mundo (Bacevich, 2009. Walt, 2010). Asimismo, señalan que esta autoimagen mítica es problemática, pues ha tenido y sigue teniendo un impacto enorme en la política exterior estadounidense, produciendo serias distorsiones en la misma, ya que impide a EE.UU. adaptarse al actual escenario internacional y defender adecuadamente sus intereses nacionales (Bacevich, 2012: 233, 234 y 238, 239).

Si bien es cierto que el realismo es antiexcepcionalista, por su rechazo del espíritu de cruzada y del mesianismo, de ahí no cabe inferir, en modo alguno, que sea antiamericano o ajeno a la cultura política americana. Hay que recordar, en este sentido, que el realismo estadounidense es marcadamente americanocéntrico, pues toda la producción científica realista americana se ha realizado pensando únicamente en los intereses de EE.UU. y, particularmente, en su seguridad nacional (Arenal, 2014: 35-49).

Aunque la tradición realista pueda ser considerada como la más influyente en la política exterior estadounidense, es indudable que no ha logrado trasladar su carácter antimesiánico a la misma más en que algunas fases concretas de la historia reciente de EE.UU., y, desde luego, no de manera completa. Aun en los periodos más realistas de la política exterior estadounidense, el excepcionalismo ha estado siempre presente en la elaboración discursiva de la misma. Así, incluso en políticas exteriores consideradas realistas como la de Richard Nixon, se puede encontrar la creencia excepcionalista como elemento inspirador del discurso presidencial.

\subsection{Neoconservadurismo}

El neoconservadurismo es un híbrido entre realismo y liberalismo internacionalista, que puede ser considerado como una doctrina autónoma dentro de la teoría de la política exterior americana ${ }^{8}$. A nuestro juicio, resulta

${ }^{8}$ El llamado neoconservadurismo es una corriente de pensamiento política nacida y desarrollada en los EE.UU., que en el ámbito de la política exterior estadounidense ha dado lugar a una doctrina dotada de una sustantividad propia (Ehrman, 1995).

Araucaria. Revista Iberoamericana de Filosofia, Politica, Humanidades y Relaciones Internacionales, año $21, \mathrm{n}^{\circ} 41$. Primer semestre de 2019. Pp. 161-185. ISSN 1575-6823 e-ISSN 2340-2199 doi: 10.12795/araucaria.2019.i41.08 
inadecuado definirlo como un subtipo de doctrina realista o de doctrina wilsoniana. Su idealismo y moralismo característicos chocan frontalmente con el principio de doble moralidad realista, mientras que su componente unilateralista y militarista colisionan de manera insalvable con el institucionalismo y el legalismo wilsonianos 9 .

Esa peculiar síntesis de fuerza y valores, de poder militar y moralismo, en la que consiste el neoconservadurismo, encuentra en el excepcionalismo una de sus claves fundamentales para interpretar y analizar el mundo y el papel que en él ha de jugar EE.UU. La concepción neoconservadora del excepcionalismo, aun teniendo ciertos elementos comunes con ambas, difiere en aspectos sustantivos de las sostenidas por realistas e internacionalistas liberales. Por ende, es posible hablar, en el plano teórico, de una concepción distintivamente neoconservadora del excepcionalismo, que los propios neoconservadores reivindican como la más fiel a la historia y tradición política estadounidense.

Para los neoconservadores poder militar y valores estadounidenses están indisociablemente unidos, es decir, el neoconservadurismo exalta ambas dimensiones del excepcionalismo americano y las sitúa, en su concepción teórica, en pie de igualdad, estableciendo una relación de complementariedad indisoluble entre ambos. Así, el poder americano ha de ejercitarse para defender los valores de la nación y son estos precisamente los que proveen de la legitimidad necesaria al ejercicio del primero: no es necesario buscar legitimidades exógenas para usar la fuerza, la defensa de los propios valores proporciona la cobertura de legitimidad suficiente para la política exterior ${ }^{10}$.

Según la doctrina neoconservadora, el poder militar americano es un instrumento útil y legítimo para exportar la democracia y los valores universales de los EE.UU. Los neoconservadores asumen, así, la visión más redencionista del excepcionalismo americano: la fuerza puede y debe ser empleada no sólo para defender la democracia allá donde esté en peligro, sino también para difundirla allá donde esté ausente mediante el cambio de régimen. En consecuencia, es del todo lógico que la superioridad militar estadounidense sea un dogma neoconservador desde el propio surgimiento del neoconservadurismo en la década de los setenta. La hegemonía americana, el mundo que EE.UU. logró construir tras la Segunda Guerra Mundial, no puede mantenerse sin la necesaria asunción de determinadas cargas y responsabilidades como la de sustentar el aparato militar más poderoso del mundo (Kagan, 2014).

\footnotetext{
${ }^{9}$ El presente trabajo disiente, por ende, de la extendida (y transversal) opinión doctrinal que considera que el neoconservadurismo es un tipo de wilsonianismo: sea 'wilsonianismo duro' (Boot, 2004: 49), 'wilsonianismo con dientes' (Mearsheimer, 2005), 'wilsonianismo renacido' (Mead, 2005: 88).

10 La interpretación neoconservadora del excepcionalismo lleva irremisiblemente a un unilateralismo doctrinal: ni el Derecho internacional ni las instituciones multilaterales pueden coartar la libertad de acción soberana de la política exterior estadounidense.
} 
Para la doctrina neoconservadora, y en este punto la coincidencia con el internacionalismo liberal sí es clara, los valores americanos, representados por la Declaración de Independencia y el ideario de los Padres Fundadores, son universales y EE.UU. es la 'primera nación universal' (Wattenberg, 1991). Si, como luego se verá, el nacionalismo aislacionista lleva al ensimismamiento y a la introversión en la política exterior, el nacionalismo neoconservador conduce a un internacionalismo unilateralista y marcadamente intervencionista que hace de la difusión de los valores americanos su principal bandera.

El neoconservadurismo, desde sus propios orígenes, siempre ha estado preocupado por la decadencia de los valores americanos en el propio seno de la nación. Para los neoconservadores, el excepcionalismo actúa como un elemento de cohesión nacional, de modo que, a su juicio, la mejor manera de revitalizar los valores americanos at home es llevar a cabo una política exterior activista, basada en los valores universales que encarna EE.UU. Así, la política exterior americana, tal y como la conciben los neoconservadores, es fundamental para fortalecer la identidad nacional estadounidense. (Kristol y Kagan, 1996: 31. En un sentido similar: Ceaser, 2013: 20). Por el contrario, una política exterior débil, moralmente relativista, renuente a usar la fuerza en defensa de los propios valores, no sólo debilita la posición de EE.UU. en el mundo, sino que pone en peligro su identidad nacional en el ámbito doméstico y, por consiguiente, el propio sistema democrático americano.

De igual modo que el internacionalismo liberal, el neoconservadurismo afirma que EE.UU. tiene la misión de transformar el mundo conforme a los valores estadounidenses, pero difiere sustancialmente del idealismo wilsoniano en los medios elegidos para tal fin: es la propia acción unilateral estadounidense, amparada en su incomparable poder militar, la que ha de obrar esa transformación (Wolf, 2003). Asimismo, hay que señalar que los neoconservadores tienen una visión diferente del mundo que habría de resultar de tal intervención transformadora americana: si el internacionalismo wilsoniano ambiciona un orden internacional regido por el multilateralismo institucionalizado y el Derecho internacional, el neoconservadurismo pretende un orden internacional hegemónico, un verdadero "proyecto imperial" basado en la denominada Pax Americana (Dorrien, 2004).

Por otro lado, la doctrina neoconservadora establece un vínculo directo entre el vigor de la democracia a escala internacional y la seguridad nacional americana. A su juicio, el 11-S puso de relieve que la falta de democracia en Oriente Medio estaba en la raíz del ataque terrorista. Por lo tanto, era preciso que el poder americano se pusiera como objetivo principal la transformación democrática de la región (Kristol y Kaplan, 2003).

El neoconservadurismo defiende, en suma, una visión excepcionalista exaltada al afirmar, con mayor énfasis que cualquier otra doctrina, la 
universalidad y superioridad de los valores estadounidenses, y la necesidad de defenderlos empleando sin reticencias ni cortapisas el poder de EE.UU.

\subsection{Aislacionismo}

Al igual que los neoconservadores, los aislacionistas conectan la dimensión material con la dimensión espiritual del excepcionalismo pero lo hacen de una manera notablemente distinta. La interpretación aislacionista del excepcionalismo hace hincapié en la geografía americana. Desde su punto de vista, el aislamiento geográfico de EE.UU. le confiere una suerte de "inmunidad estratégica" (Nau, 2002: 45) que le permite mantener su independencia, garantizar su seguridad nacional y preservar intactos sus valores primigenios. En el pensamiento aislacionista, el sistema político americano sólo permanecerá incólume manteniéndose al margen de un Viejo Mundo marcado por la anarquía y el conflicto (Nau, 2002: 45) Por consiguiente, los aislacionistas, en claro contraste con internacionalistas liberales y neoconservadores, interpretan el excepcionalismo americano en términos de insularidad, no de universalidad.

El aislacionismo opta por la separación del Viejo Mundo para preservar el Nuevo Mundo (Anderson, 2014: 13), de modo que el sistema sociopolítico de EE.UU. sea un ejemplo a seguir para el resto de naciones. Para los aislacionistas la misión estadounidense en el mundo se limita, por lo tanto, a predicar con su poderoso ejemplo, esto es, al 'ejemplarismo'. Por consiguiente, EE.UU. no tiene la misión de promover activamente la democracia, ni de ser el sheriff del mundo, sino que sólo debe intervenir militarmente fuera de sus fronteras, dentro de un estricto sentido de autodefensa, cuando la seguridad americana se vea directamente comprometida (Nordlinger, 1995).

Los aislacionistas comparten la descripción realista del mundo: un mundo hobbesiano marcado por el conflicto y carente de una autoridad central. Sin embargo, su respuesta ante éste es diferente, mientras que los realistas eligen, como se ha visto, intervenir y participar de lleno en los asuntos internacionales para defender el interés nacional y garantizar la seguridad nacional americana, los aislacionistas consideran que la intervención americana en los asuntos internacionales, especialmente en lo que concierne al uso de la fuerza, ha de ser mínima (Nau, 2002, 45) para evitar sus inevitables consecuencias negativas.

Al igual que el neoconservadurismo, el aislacionismo es fuertemente unilateralista y, en consecuencia, rechaza las alianzas con Europa y Asia al entender que limitan la acción estadounidense e involucran al país en problemas ajenos a su interés nacional (Nau, 2002: 44). Ahora bien, a diferencia del unilateralismo neoconservador, que es marcadamente intervencionista y misionero, el unilateralismo aislacionista es profundamente introvertido, 
refractario al aventurismo militar y contrario a los proyectos mesiánicos para transformar el mundo.

En la Posguerra Fría el discurso aislacionista supone renunciar tanto a intervenciones humanitarias ajenas al interés de EE.UU. como a proyectos mesiánicos dirigidos a transformar democráticamente el mundo, particularmente Oriente Medio. La seguridad nacional estadounidense no está vinculada en modo alguno a la expansión mundial de la democracia, sino que la misma puede garantizarse perfectamente al margen de cuál sea la forma de gobierno elegida por el resto de Estados (Nordlinger, 1995. Nau, 2002: 45). Por lo tanto, el aislacionismo no sigue la idea de paz democrática abrazada (si bien de modos distintos) por internacionalistas liberales y neoconservadores.

Para los aislacionistas, la política exterior mesiánica y militarista de George W. Bush es una política imperialista completamente rechazable, pues el imperialismo lleva a la destrucción de la idea americana y a la propia ruina económica y moral de EE.UU. Ello explica que los aislacionistas fueran uno de los sectores conservadores más combativos contra la decisión mayoritaria dentro del conservadurismo americano y del Partido Republicano de llevar a cabo el cambio de régimen en Irak (Buchanan, 2005 : 11-13).

Por otro lado, hay que señalar que el aislacionismo se ha quedado fuera de los grandes debates existentes en el seno del Partido Republicano y del conservadurismo americano por el control de la política exterior. La ascensión de EE.UU. a superpotencia y líder del bloque occidental como resultado de la Segunda Guerra Mundial hizo inviable todo retroceso a las políticas del siglo XIX y del periodo de entreguerras del siglo XX (bien entendido que se trataba de un aislamiento hemisférico, en absoluto de inacción o aislamiento totales). Las capacidades económicas y militares de EE.UU., así como la vigencia del excepcionalismo redencionista, imposibilitan la aplicación práctica de los postulados aislacionistas.

Ahora bien, el hecho de que el aislacionismo haya dejado, como tal, de ser una opción doctrinal aplicable a la política exterior estadounidense no significa que la idea de insularidad no siga teniendo un fuerte predicamento en la opinión pública estadounidense. Asimismo, el aislacionismo sigue muy presente en la contienda ideológica en torno a la política exterior, pues suele ser invocado por los partidarios de otras doctrinas, particularmente la neoconservadora, como una amenaza para la primacía americana y para la seguridad nacional ${ }^{11}$.

\footnotetext{
${ }^{11}$ Los neoconservadores emplean el término 'aislacionista' para descalificar una política exterior reacia a la intervención militar, o a políticos que priorizan la política interior sobre la exterior. En estos casos, el término se emplea de manera peyorativa e hiperbólica, con finalidad de exaltar la batalla político-ideológica.
}

Araucaria. Revista Iberoamericana de Filosofia, Politica, Humanidades y Relaciones Internacionales, año $21, \mathrm{n}^{\circ} 41$. Primer semestre de 2019. Pp. 161-185. ISSN 1575-6823 e-ISSN 2340-2199 doi: 10.12795/araucaria.2019.i41.08 


\section{El excepcionalismo en la política exterior de EE.UU. del siglo XXI}

El inicio del siglo XXI permite comprender la importancia del excepcionalismo en la política exterior estadounidense. No se entrará a considerar si el excepcionalismo es un mito o una realidad: no importa tanto que EE.UU. sea o siga siendo cualitativamente diferente al resto del mundo, sino que esta idea permanece viva en la sociedad y en la política americanas.

El periodo que comprende las dos presidencias concluidas en lo que llevamos de siglo XXI permite observar perfectamente que el excepcionalismo mantiene una fuerza enorme en EE.UU. y que últimamente ha estado en el centro de los debates en torno a la orientación de la política exterior de la superpotencia. Asimismo, este periodo pone de relieve que el excepcionalismo carece también en la práctica política de una interpretación y aplicación únicas, y que admite diversos e importantes matices dentro de la adhesión a un núcleo de ideas comunes.

\subsection{De Bush a Obama: la nueva crisis del excepcionalismo}

La política exterior de la Administración Bush, particularmente durante su primer mandato, no puede ser entendida sin el excepcionalismo americano, en concreto, sin la interpretación neoconservadora del mismo. Alineado con los postulados neoconservadores, George W. Bush ha protagonizado el revival excepcionalista más potente desde la era Reagan.

La política de Bush, tras el 11-S y como consecuencia directa del mismo, operó una serie de cambios sustanciales que quedaron plasmados en la National Security Strategy (NSS) de septiembre de 2002 (Bush, 2002). La NSS ha de ser considerada como un documento inequívocamente excepcionalista y, en concreto, marcadamente redencionista o vindicacionista (Monten, 2005: 113). Bush asume la idea excepcionalista de que EE.UU. es el agente de transformación y progreso más importante del mundo: posee unos valores universales y un poder militar inigualable necesario para defenderlos y difundirlos (Bush, 2002: 1 y 3). Por ello, hace suya la idea neoconservadora de la necesidad de democratizar Oriente Medio mediante el cambio de régimen para garantizar la seguridad nacional, eleva a doctrina las acciones militares preventivas y decide que EE.UU. puede actuar militarmente sin las constricciones derivadas de las instituciones internacionales y del Derecho internacional (Bush, 2002: 6 y 15).

La presidencia de Bush expira con el país sumido en la crisis económica y financiera más grave desde el Great Crash de 1929 y con el peso de haber embarcado al país en una guerra imposible de ganar como la de Irak. Asimismo, la política exterior de Bush ha mostrado las consecuencias contraproducentes del excepcionalismo. Si el excepcionalismo posee un "magnetismo" capaz de atraer 
tanto a otros Estados hacia la órbita de EE.UU. como a seres humanos de todo el mundo en busca del 'sueño americano', no es menos cierto que también genera un fuerte rechazo en forma de antiamericanismo (Dunn, 2013: 3 y 6). En el caso de Bush, su política exterior generó la mayor ola de antiamericanismo desde el final de la Guerra Fría. En este contexto, la llegada de Obama al poder se plantea en términos de cambio, tanto en política interna como en política exterior.

\subsection{Obama y el excepcionalismo americano}

La presidencia de Obama ha desatado un debate de gran magnitud en torno al excepcionalismo americano prácticamente desde su llegada al poder. La cuestión que se plantea es si Obama ha abandonado la creencia excepcionalista acogiéndose a otras ideas ajenas a la tradición política estadounidense. Dicho en otros términos: ¿ha sido la política exterior de Obama excepcionalista?

Según sus críticos, la respuesta a esta interrogante es clara: Obama es un relativista moral, que no cree en la superioridad de los valores americanos. En otros términos, Obama no cree que EE.UU. sea una "fuerza para el bien"12 en el mundo. Ello supone, a su vez, la incapacidad para distinguir entre los amigos y los enemigos de EE.UU., llevándole a pactar con los últimos y a abandonar a su suerte a los primeros (Muravchik, 2010). La falta de creencia en la superioridad y universalidad de los valores americanos supone la incapacidad para defenderlos e impulsar su avance, de ahí que Obama haya recortado también el gasto militar, dañando gravemente el poder americano.

Para los neoconservadores, el declive estadounidense en el mundo no es una realidad objetiva e inevitable, sino que son las políticas antiexcepcionalistas como las de Obama las que conducen al mismo. Por ende, está en manos únicamente de EE.UU. evitar su declive y continuar siendo la superpotencia hegemónica y el líder del mundo libre también durante el siglo XXI (Krauthammer, 2009). Según Kagan, Obama, con el apoyo de la mayoría de los estadounidenses, no sólo lleva a cabo un repliegue coyuntural, sino que encarna la idea de la dejación de las responsabilidades globales de EE.UU., una idea extremadamente peligrosa pues su puesta en práctica puede tener consecuencias irreversibles (Kagan, 2014).

Las críticas contra Obama pretenden situar al presidente americano en el entorno ideológico de las doctrinas anti-imperialistas, absolutamente marginales en EE.UU., o bien cercano a la izquierda socialdemócrata europea. De este modo se ha querido presentar a Obama como un presidente extranjero con ideas extrañas a las tradiciones políticas y culturales americanas (Montopoli, 2010). Por ello, no puede sorprender que Obama haya sido tachado de antiexcepcionalista y antiamericano, e incluso, que desde sectores del llamado

12 A force for good, en la expresión original en inglés

Araucaria. Revista Iberoamericana de Filosofia, Politica, Humanidades y Relaciones Internacionales, año $21, \mathrm{n}^{\circ} 41$. Primer semestre de 2019. Pp. 161-185. ISSN 1575-6823 e-ISSN 2340-2199 doi: 10.12795/araucaria.2019.i41.08 
Tea Party, se haya cuestionado su propia americanidad, poniendo en duda su nacionalidad, a fin socavar su propia legitimidad como presidente (Tashman, 2011).

El tono y contenido exacerbados de estas críticas dificulta el propio entendimiento del excepcionalismo y de sus distintas interpretaciones. Tal exacerbación responde a la pretensión de determinados sectores políticoideológicos, como los neoconservadores, de apropiarse del excepcionalismo americano y de imponer su visión del mismo como la única posible y existente en la actualidad, rechazando de plano otras interpretaciones alternativas.

El discurso del presidente Obama sobre el excepcionalismo en materia de política exterior ha sido contradictorio. Si en Estrasburgo, en el marco de la cumbre de la OTAN, venía a negar la propia esencia del excepcionalismo americano al afirmar que EEUU es tan excepcional como cualquier otra nación (Obama, 2009b), en intervenciones posteriores se ha declarado fervientemente excepcionalista (v. gr.: Obama, 2014).

El estudio de tres casos que han tenido un papel central en la política exterior de la Administración Obama nos permite elucidar adecuadamente su visión del excepcionalismo americano y enmarcarla en las distintas visiones analizadas: su política de apertura hacia Cuba; las negociaciones que han dado lugar un acuerdo internacional sobre el programa nuclear de Irán; y su política en la guerra de Siria. No se trata aquí de analizar en profundidad estas políticas y si las mismas han sido o no exitosas, sino sólo de limitarnos a descifrar qué concepción del excepcionalismo americano subyace en las mismas.

En cuanto a Cuba, Obama ha pretendido acabar con la idea de que todo cambio de la política de EE.UU. hacia la isla había de estar condicionado a la previa caída del régimen castrista y al inicio de la democratización cubana. Obama ha abandonado, así, el maximalismo excepcionalista de exigir un nuevo régimen democrático en La Habana como conditio sine qua non para empezar a levantar el embargo. El inicio de esta política de apertura en diciembre de 2014, conducente a desmantelar la histórica política de bloqueo estadounidense a Cuba, ha de enmarcase en el cambio que ha querido aplicar EE.UU. respecto a Latinoamérica desde que el Secretario de Estado John Kerry proclamara el fin de la tradicional aproximación injerencista hacia el subcontinente, la célebre doctrina Monroe (Reid, 2015). De hecho, la nueva política hacia Cuba desarrollada en sus dos últimos años en la Casa Blanca ha sido el cambio más sustantivo, la punta de lanza, de la política exterior latinoamericana de EE.UU. durante los dos mandatos de Obama (Gillin, 2015).

En lo referente a Irán, la constante voluntad de llegar a un acuerdo con el régimen de los ayatolás en torno a su programa nuclear y la culminación de las negociaciones, junto con las grandes potencias del Consejo de Seguridad y la Unión Europea, en Viena en julio de 2015, sitúan a Obama muy alejado de las 
posiciones excepcionalistas de corte más vindicacionista. Para éstas no ya la posibilidad de un pacto sino la propia idea de negociación con un régimen como el iraní es, en sí, indeseable; por ello no es de extrañar que hayan denunciado en todo momento el proceso negociador con Irán, equiparándolo al Pacto de Múnich de 1938 entre Chamberlain y Hitler (DelReal, 2013). La propia naturaleza tiránica de la República Islámica de Irán y su declarada intención de destruir Israel hacen indeseable alcanzar cualquier tipo de acuerdo con Teherán. Hacerlo supone claudicar en el defensa de los valores fundacionales americanos y ceder ante un enemigo que busca la destrucción de los mismos (Ledeen, 2009: 4; Bolton, 2015).

La controvertida política de Obama hacia la guerra civil siria y su decisión de no intervenir militarmente contra el régimen presidido por Bashar al-Assad, pese a haber traspasado las propias líneas rojas fijadas por EE.UU., constituyen otro claro ejemplo de cómo concibe Obama el excepcionalismo americano. Esta política ha de ser encuadrada en el abandono de los proyectos mesiánicos de transformación democrática de Oriente Medio con el consiguiente rechazo a la idea de democratizar la región mediante el cambio de régimen y el nation building (Lynch, 2015). La política exterior de Obama hacia Oriente Medio ha contrastado claramente con el mesianismo que impregnó la misma durante el primer mandato de George W. Bush. Obama ha querido articular una política exterior consistente en "liderar desde atrás" (Singh: 39-48) ${ }^{13}$, evitando la intervención militar directa de EE.UU. tanto en Siria como en el resto de la región.

En estos tres casos expuestos, se pone claramente de relieve que Obama ha impugnado las visiones más duras del excepcionalismo que abogan tanto por un protagonismo claro de EE.UU. en la promoción mundial de la democracia como por la oposición a dialogar y alcanzar acuerdos con las tiranías. Obama ha rechazado, así, el espíritu misionero propio del redencionismo, introduciendo un importante componente de autocrítica y ha abandonado expresamente la política de democratización manu militari, en línea con su idea de que la democracia no puede ser impuesta desde fuera (Obama, 2009a). Asimismo, estos tres casos permiten afirmar que respecto del excepcionalismo Obama se ha movido entre dos órbitas doctrinales. En primer lugar, dentro de la órbita realista, su admiración hacia Reinhold Niebuhr ha quedado patente en su consciencia de que EE.UU. no está exento de incurrir en los vicios de cualquier otra nación y que debe huir de la arrogancia imperial (Holder y Josephson, 2015. Douthat, 2015). Ello supone un cuestionamiento del excepcionalismo entendido en términos de fuente de legitimidad para imponer los valores americanos en el exterior. En segundo lugar, Obama se ha movido también en la órbita natural del Partido Demócrata, esto es, la del internacionalismo

\footnotetext{
${ }^{13}$ Leading from behind, en la expresión original en inglés.
} 
liberal, encontrándose mucho más próximo a la línea globalista que a la intervencionista. Ello se ha visto sobre todo reflejado en su preferencia por la diplomacia multilateral para resolver conflictos como el iraní y en el apoyo a la gobernanza global en materia de desarme nuclear ${ }^{14}$.

En consecuencia, Obama, lejos de ser un antiexcepcionalista radical, como pretenden sus más acerbos críticos, se ha movido en posiciones excepcionalistas moderadas siguiendo las sendas del realismo niebuhriano y del globalismo liberal. Obama no deja de creer en la diferencia y unicidad de EE.UU. pero se opone a su papel como redentor del mundo en los términos establecidos por versiones más duras del excepcionalismo.

\section{Conclusiones}

A lo largo de este trabajo se ha podido comprobar que no existe en EE.UU. una concepción unívoca y monolítica del excepcionalismo americano. Tras un periodo de reafirmación y expansión en sus términos más duros, la política exterior americana ha basculado hacia posiciones de un relativo repliegue internacional y hacia la atenuación significativa, que no abandono, del discurso excepcionalista.

Obama considera que una política exterior como la del primer mandato de George W. Bush, basada en una concepción redencionista dura del excepcionalismo, sólo puede arruinar el prestigio de EE.UU. y cercenar su capacidad de liderazgo global. Aplicando dosis de realismo nieburhriano y neorrealismo defensivo, Obama ha tratado de resolver los problemas creados por la política primacista y redencionista de su predecesor; asimismo, a través de su apuesta por el multilateralismo institucionalizado para tratar de gobernar la globalización ha buscado restaurar la imagen y el prestigio internacional de EE.UU. Para sus críticos, en cambio, es la política de Obama, marcada por la debilidad, la que conduce a la retirada del mundo, al declive internacional de EE.UU. y, finalmente, al ocaso del orden mundial americano.

El excepcionalismo se ha convertido en motivo de querella política entre los dos grandes partidos estadounidenses. Sólo desde intereses partidistas exacerbados, y en el marco de la polarización que domina la política estadounidense, es sostenible afirmar que Obama sea antiexcepcionalista y, más aún, antiamericano. Antes bien, en materia de política exterior, Obama ha de ser considerado como un excepcionalista tibio o moderado, que viene transitando entre la autocrítica moralista niebuhriana y el redencionismo suave

${ }^{14}$ Otro ámbito en el que se observa con claridad el globalismo de Obama ha sido su promoción de la gobernanza global en la lucha contra el cambio climático, culminada con la ratificación del Acuerdo de París en septiembre de 2016. 
propio de los globalistas.

El excepcionalismo es una idea profundamente arraigada en la cultura política y en la identidad nacional estadounidenses. Se ha comprobado que, salvo el notable caso del realismo, todas las doctrinas estadounidenses abrazan de una forma u otra la idea excepcionalista. Ni la derrota en Vietnam, que demostró que EE.UU. no era invencible, ni el 11-S, que constató que el territorio continental americano era vulnerable, seguido del fracaso en la guerra de Irak, han supuesto el fin del excepcionalismo estadounidense. Éste seguirá jugando un papel importante en la concepción e implementación de la política exterior americana y continuará presente tanto en los debates teóricos en torno a la misma como en su práctica, especialmente en el discurso presidencial.

Asimismo, no es posible ignorar que el excepcionalismo sigue teniendo una importancia fundamental en la política americana y en su política exterior en particular: la de la búsqueda de la legitimidad de ejercicio. Ningún presidente americano puede definirse públicamente como antiexcepcionalista, ni actuar abiertamente como tal, sin soportar unos costes inasumibles. Por eso todos los presidentes americanos, incluso los considerados realistas, han invocado la creencia excepcionalista en sus discursos presidenciales. Obama no ha sido una excepción a esta regla. 


\section{Referencias bibliográficas:}

Anderson, Perry. 2014. Imperium et Consilium: la politica exterior norteamericana y sus teóricos, trad. de Jaime Blasco, Madrid: Akal.

Arenal, Celestino del. 2014. Etnocentrismo y teoría de las relaciones internacionales: una visión crítica, Madrid: Tecnos.

Arenal, Celestino del. 1990.: Introducción a las relaciones internacionales, $3^{\mathrm{a}}$ ed., Madrid: Tecnos.

Bacevich, Andrew J. (ed.). 2012. The Short American Century: A Postmortem, Cambridge: Harvard University Press.

Bacevich, Andrew J. 2012. "Not So Different After All" en Bacevich, Andrew J. (ed.) (2012): The Short American Century: A Postmortem, Cambridge: Harvard University Press, pp. 231-239.

Bacevich, Andrew J. 2009. The Limits of Power: The End of American Exceptionalism, New York: Holt Paperbacks.

Barone, Michael. 2013. "Restoring Reaganesque Exceptionalism in American Politics" en Dunn, Charles W. (ed.): American Exceptionalism: the origins, history, and future of the nation's greatest strength, Lanham: Rowman \& Littlefield Publishers, pp. 143-150.

Bolton, John R. 2015. “To Stop Iran's Bomb, Bomb Iran”, The New York Times, March 26, en: https://www.nytimes.com/2015/03/26/opinion/to-stopirans-bomb-bomb-iran.html? $\mathrm{r}=0$

Boot, Max. 2004. "Myths about Neoconservatism" en Stelzer, Irwin (2004): Neoconservatism, London: Atlantic Books, pp. 43-52.

Brooks, Stephen. 2013. American Exceptionalism in the Age of Obama, New York: Routledge.

Buchanan, Patrick J. 2005. Where the Right Went Wrong: How the Neoconservatives Subverted the Reagan Revolution and Hijacked the Bush Presidency, New York: Thomas Dunne Books.

Bush, George W. 2002. The National Security Strategy of the United States of America, Washington, DC: The White House, September.

Ceaser, James W. 2013. "The Origins and Character of American Exceptionalism" en Dunn, Charles W. (ed.): American Exceptionalism: the origins, history, and future of the nation's greatest strength, Lanham: Rowman \& Littlefield Publishers, pp. 11-26.

Delreal, Jose. 2013. "Charles Krauthammer: Worst since Munich”, Politico, 26 de noviembre de 2013, en: http://www.politico.com/story/2013/11/ charles-krauthammer-obama-munich-100383.html

Douthat, Ross. 2015. "Obama the Theologian”, The New York Times, February 7, en: http://www.nytimes.com/2015/02/08/opinion/sunday/ross-douthatobama-the-theologian.html?_r=0 
Dorrien, Gary. 2004. Imperial Designs: Neoconservatism and the New Pax Americana, New York: Taylor \& Francis Books.

Dunn, Charles W. (ed.). 2013. American Exceptionalism: The Origins, History, and Future of the Nation's Greatest Strength, Lanham: Rowman \& Littlefield.

Dunn, Charles W. 2013. "The Magnetism of American Exceptionalism" en Dunn, Charles W. (ed.) 2013. American Exceptionalism: The Origins, History, and Future of the Nation's Greatest Strength, Lanham: Rowman \& Littlefield, pp. 1-7.

Ehrman, John. 1995. The Rise of Neoconservatism: Intellectuals and Foreign Affairs, 1945-1994, New Haven: Yale University Press.

Fonte, John. 2012. Sovereignty or Submission: Will Americans Rule Themselves or be Ruled by Others?, New York: Encounter Books.

Gillin, Joel. 2015. "The Cuban Thaw Is Obama's Finest Foreign Policy Achievement to Date", New Republic, April 13, en: https://newrepublic. com/article/121522/cuba-obamas-biggest-foreign-policy-success

Heclo, Hugh. 2013. "Varieties of American Exceptionalism" en Dunn, Charles W. (ed.): American Exceptionalism: the origins, history, and future of the nation's greatest strength, Lanham: Rowman \& Littlefield Publishers, pp. 27-40.

Hodgson, Godfrey. 2009. The Myth of American Exceptionalism, New Haven, London: Yale University Press.

Holder, R. Ward and Josephson, Peter B. 2012. The Irony of Barack Obama: Barack Obama, Reinhold Niebuhr and the Problem of Christian Statecraft, Burlington: Ashgate.

Hayward, Steven F. 2013. "Back to the Future: Ronald Reagan Exceptionalism", en Dunn, Charles W. (ed.): American Exceptionalism: the origins, history, and future of the nation's greatest strength, Lanham: Rowman \& Littlefield, pp. 151-160.

Ignatieff, Michael (ed.). 2005. American Exceptionalism and Human Rights, Princeton: Princeton University Press.

Ignatieff, Michael. 2005. "Introduction: American Exceptionalism and Human Rights” en Ignatieff, Michael (ed.) 2005. American Exceptionalism and Human Rights, Princeton: Princeton University Press, pp. 1-26.

Kagan, Robert. 2014. "Superpowers Don't Get to Retire”, The New Republic, May 26, en: http://www.newrepublic.com/article/117859/allurenormalcy-what-america-still-owes-world

Krauthammer, Charles. 2009. "Decline is a Choice: The New Liberalism and the end of American ascendancy", The Weekly Standard, October 19, Vol. 15, No. 05, en: http://www.weeklystandard.com/Content/Public/ Articles/000/000/017/0561fnpr.asp 
Kissinger, Henry. 2010. [1994]: Diplomacia, trad. de Mónica Utrilla, Barcelona: Ediciones B.

Knock, Thomas J. 1995. To End All Wars: Woodrow Wilson and the Quest for a New World, Princeton: Princeton University Press.

Kristol, William and Kagan, Robert. 1996. "Toward a Neo-Reaganite Foreign Policy", Foreign Affairs, Vol. 75, Issue 4, July/August, pp. 18-32.

Kristol, William y Kaplan, Lawrence F. 2003. The War over Iraq: Saddam's Tyranny and America's Mission, New York: Encounter Books.

Ledeen, Michael A. 2009. Accomplice to Evil: Iran and the War Against the West, New York, St.: Martin's Press.

Lipset, Seymour Martin. 2000. [1996]: El excepcionalismo norteamericano: una espada de dos filos, México D.F.: Fondo de Cultura Económica.

Lynch, Marc. 2015. "Obama and the Middle East”, Foreign Affairs, September/ October, pp. 18-27.

Mead, Walter Russell. 2005. Power, Terror, Peace, and War: America's Grand Strategy in a World at Risk, New York: Vintage Books.

Mearsheimer, John. 2005. "Hans Morgenthau and the Iraq war: realism versus neo-conservatism", Open Democracy, April 21, en: https://www. opendemocracy.net/democracy-americanpower/morgenthau_2522.jsp

McCrisken, Trevor B. 2003. American Exceptionalism and the Legacy of Vietnam: US Foreign Policy since 1974, New York: Palgrave MacMillan.

Monten, Jonathan. 2005. "The Roots of the Bush Doctrine: Power, Nationalism, and Democracy Promotion in U.S. Strategy", International Security, 29 (4), pp. 112-156.

Montopoli, Brian. 2010. "Sarah Palin: Obama's Policies are Un-American", CBS News, April 14, en: http://www.cbsnews.com/news/sarah-palinobamas-policies-are-un-american/

Morgenthau, Hans J. 1986. [1948]: Política entre las naciones. La lucha por el poder y la paz, Buenos Aires: Grupo Editor Latinoamericano.

Muravchik, Joshua. 2010. Obama's Radical Transformation of America: Year One, New York: Encounter Books.

Nau, Henry N. 2002. At Home Abroad: Identity and Power in American Foreign Policy, Ithaca: Cornell University Press.

Nordlinger, Eric. 1995. Isolationism Reconfigured, Princeton: Princeton University Press.

Obama, Barack. 2014. "Remarks by the President at the United States Military AcademyCommencementCeremony",U.S.MilitaryAcademy-WestPoint, West Point, New York, May 28, en: https://www.whitehouse.gov/ the-press-office/2014/05/28/remarks-president-united-states-militaryacademy-commencement-ceremony 
Obama, Barack. 2009a. "Remarks by the President at Cairo University", June

4, en: https://www.whitehouse.gov/the-press-office/remarks-presidentcairo-university-6-04-09

Obama, Barack. 2009b. "News conference by President Obama", Strasbourg, France, The White House, April 4, en: http://www.whitehouse.gov/thepress-office/news-conference-president-obama-4042009

Reid, Michael. 2015. "Obama and Latin America”, Foreign Affairs, September/ October, pp. 45-53.

Rosen, Gary (ed.). 2005. The Right War?: The Conservative Debate On Iraq, New York: Cambridge University Press.

Söderlind, Sylvia and Carson, James Taylor (eds.). 2011. American Exceptionalisms: from Winthorp to Winfrey, Albany: State University of New York Press.

Spanier, John. 1991. La política exterior norteamericana a partir de la Segunda Guerra Mundial, trad. de Cristina Piña, Buenos Aires: Grupo Editor Latinoamericano.

Stephanson, Anders. 1996. Manifest Destiny: American Expansion and the Empire of Right, New York: Hill \& Wang.

Tashman, Brian. 2011. "Tea Party Nation goes Birther", Right Wing Watch, July, 8, en: http://www.rightwingwatch.org/post/tea-party-nation-goes-birther/

Thompson, Michael G. 2007. "An Exception to Exceptionalism: A Reflection on Reinhold Niebuhr's Vision of "Prophetic" Christianity and the Problem of Religion and U.S. Foreign Policy", American Quarterly, vol. 59, number 3, September, pp. 833-855.

Tocqueville, Alexis de. 2002. [1840]: La democracia en América, 2, Madrid: Alianza Editorial.

Tovar Ruiz, Juan. 2014. "El idealismo wilsoniano en la política exterior estadounidense, ¿una doctrina recurrente?", Revista Española de Ciencia Politica, núm. 35, julio, pp. 137-160.

Walt, Stephen M. 2011. "The Myth of American Exceptionalism", Foreign Policy, October 11, en: http://foreignpolicy.com/2010/12/06/americanexceptionalism-a-realist-view/

Walt, Stephen M. 2010. “American Exceptionalism: a realist view”, Foreign Policy, December 6, en: http://foreignpolicy.com/2010/12/06/americanexceptionalism-a-realist-view/

Wattenberg, Ben J. 1991. The First Universal Nation. Leading Indicators and Ideas about the Surge of America in the 1990's, New York: Free Press.

Weinberg, Albert K. 1968 [1935]: Destino manifiesto: el expansionismo nacionalista en la historia norteamericana, Buenos Aires: Paidós.

Wolf, Martin. 2003. "Bush is all big stick and no soft speech", Financial Times, December 24. 
